\title{
Root Cube Mean Cordial Labeling of Some Standard Graphs
}

\author{
N. G. Gidheeba ${ }^{1}$ and A. Vijayan ${ }^{2}$ \\ ${ }^{1}$ Ph.D. Research Scholar (Part time), Register Number : 18233112092024, Nesamony \\ Memorial Christian College, Marthandam, Kanyakumari District, Tamil Nadu, India \\ 2Research Department of Mathematics, Nesamony Memorial Christian College, \\ Marthandam, Kanyakumari District, Tamil Nadu, India
}

\section{ABSTRACT}

Let $\mathrm{G}=(\mathrm{V}, \mathrm{E})$ be a graph and $\mathrm{f}$ be a mapping from $\mathrm{V}(\mathrm{G}) \rightarrow\{0,1,2\}$. For each edge uv of $\mathrm{G}$ assign the label $\left\lfloor\sqrt{\frac{\sqrt{(\mathrm{u}))^{3}+\mathrm{f}(\mathrm{v})^{3}}}{2}} \mid\right.$. $\mathrm{f}$ is called a root cube mean cordial labeling if $\left.\right|_{\mathrm{vf}}(\mathrm{i})-_{\mathrm{vf}}(\mathrm{j}) \mid$ ET1 and $\left.\right|_{\mathrm{ef}}(\mathrm{i})-_{\mathrm{ef}}(\mathrm{j}) \mid \leq 1$, where ${ }_{\mathrm{vf}}(\mathrm{x})$ and ${ }_{\mathrm{ef}}(\mathrm{x})$ denote the number of vertices and edges labeled with $x, x \in\{0,1,2\}$ respectively. A graph with a root cube mean cordial labeling is called root cube mean cordial graph . In this paper, root cube mean cordiality of some standard graphs such as cycle, path, star are discussed .

KEY WORDS: CYCLE, PATH, ROOT CUBE MEAN CORDIAL LABELING, ROOT CUBE MEAN CORDIAL GRAPHS.

\section{INTRODUCTION}

The graphs considered here are finite, undirected and simple. The vertex set and edge set of a graph $G$ are denoted by $V(G)$ and $E(G)$ respectively. Labeled graphs are useful models for a broad range of applications such as coding theory, $\mathrm{X}$ - ray crystallography, astronomy, circuit design etc. The concept of cordial labeling was introduced by Cahit in the year 1987. Let $\mathrm{f}: \mathrm{V}(\mathrm{G}) \leq\{0,1\}$ be a function. For each edge uv assign the label $\mid \mathrm{f}(\mathrm{u})-$ $f(v) \mid$. $f$ is called a cordial labeling if $\left|{ }_{v f}(i)-{ }_{v f}(j)\right| \leq 1$ and $\left|\operatorname{ef}(\mathrm{i})-_{\mathrm{EF}}(\mathrm{j})\right| \leq 1, \mathrm{i}, \mathrm{j} \leq\{0,1\}$ where $\mathrm{vf}(\mathrm{x})$ and $\mathrm{ef}(\mathrm{x})$ denote the number of vertices and edges respectively labeled with $\mathrm{x}(\mathrm{x}=0,1)$.A graph which admits a cordial labeling is called cordial graph.

\section{ARTICLE INFORMATION}

Received 8th Oct 2020 Accepted after revision 25th Dec 2020 Print ISSN: 0974-6455 Online ISSN: 2321-4007 CODEN: BBRCBA

Thomson Reuters ISI Web of Science Clarivate Analytics USA and Crossref Indexed Journal

\section{Clarivate
Analytics}

NAAS Journal Score 2020 (4.31)

A Society of Science and Nature Publication,

Bhopal India 2020. All rights reserved.

Online Contents Available at: http//www.bbrc.in/

Doi: http://dx.doi.org/10.21786/bbrc/13.15/58

\section{Root Cube Mean Cordial Labeling Definition 2.1}

Let $\mathrm{G}=(\mathrm{V}, \mathrm{E})$ be a graph and $\mathrm{f}$ be a mapping from $\mathrm{V}(\mathrm{G}) \rightarrow\{$ $0,1,2\}$. For each edge uv of $\mathrm{G}$ assign the label

$$
\left\lfloor\sqrt{\frac{f(u)^{3}+f(v)^{3}}{2}}\right\rfloor
$$

$\mathrm{f}$ is called a root cube mean cordial labeling if $\mid \mathrm{vf(i)}$ $\operatorname{vf(j)} \mid \leq 1$ and $\mid$ ef(i) $-\operatorname{ef(j)} \mid \leq 1$, where vf(x) and ef(x) denote the number of vertices and edges labeled with $\mathrm{x}, \mathrm{x} \varepsilon\{0,1,2\}$ respectively, A graph with a root cube mean cordial labeling is called root cube mean cordial graph. We illustrate the definition using the following example.

Example 2.2 A graph that admits a root cube mean cordial labeling is given below.

Here $\mathrm{v}_{\mathrm{f}}(\mathrm{o})=2, \operatorname{vf}(1)=3, \operatorname{vf}(2)=3$ and $\operatorname{ef}(0)=3, \operatorname{ef}(1)=2$, ef(2) $=3$ and $\left|v f(i)-v_{f}(j)\right| \leq 1$ and $\mid$ ef(i) $-\operatorname{ef}(j) \mid \leq 1$ for all $i, j \varepsilon\{0,1,2\}$. 
Theorem 2.3. The graph $\mathrm{Pn}$ is a root cube mean cordial graph.

\section{Proofs}

Let $P_{n}$ be the path $u_{1} u_{2} \ldots u_{n}$ where $\left|V\left(P_{n}\right)\right|=n$

and $\left|\mathrm{E}\left(\mathrm{P}_{\mathrm{n}}\right)\right|=\mathrm{n} \square 1$.

Define $f: V\left(P_{n}\right) \rightarrow\{0,1,2\}$ as follows.

Case (i) $n=0(\bmod 3)$

Let $\mathrm{n}=3 \mathrm{t}$, Define $\mathrm{f}\left(\mathrm{u}_{\mathrm{i}}\right)=0,1 \leq \mathrm{i} \leq \mathrm{t}$

$$
\begin{aligned}
& \mathrm{f}\left(\mathrm{u}_{\mathrm{t}+\mathrm{i}}\right)=1,1 \leq \mathrm{i} \leq \mathrm{t} \\
& \mathrm{f}\left(\mathrm{u}_{2 \mathrm{t}-\mathrm{i}}\right)=2,1 \leq \mathrm{i} \leq \mathrm{t}
\end{aligned}
$$

Then $v_{\mathrm{f}}(0)=\mathrm{t}, \mathrm{v}_{\mathrm{f}}(1)=\mathrm{t}, \mathrm{v}_{\mathrm{f}}(2)=\mathrm{t}$

$$
\mathrm{e}_{\mathrm{f}}(0)=\mathrm{t}, \mathrm{e}_{\mathrm{f}}(1)=\mathrm{t} \square 1, \mathrm{e}_{\mathrm{f}}(2)=\mathrm{t} \text {. }
$$

Therefore, $\left|\mathrm{v}_{\mathrm{f}}(\mathrm{i})-\mathrm{v}_{\mathrm{f}}(\mathrm{j})\right| \leq 1$ and $\left|\mathrm{e}_{\mathrm{f}}(\mathrm{i})-\mathrm{e}_{\mathrm{f}}(\mathrm{j})\right|$ for all $i, j \in\{0,1,2\}$.

Hence $P_{n}$ is a root cube mean cordial graph.

Case (ii) $\mathrm{n} \equiv 1(\bmod 3)$

Let $\mathrm{n}=3 \mathrm{t}+1$. Define $\mathrm{f}\left(\mathrm{u}_{\mathrm{i}}\right)=0,1 \leq \mathrm{i} \leq \mathrm{t}$

$$
\begin{aligned}
& \mathrm{f}\left(\mathrm{u}_{\mathrm{t}-\mathrm{i}}\right)=1,1 \leq \mathrm{i} \leq \mathrm{t}+1 \\
& \mathrm{f}\left(\mathrm{u}_{2 \mathrm{t}-1-\mathrm{i}}\right)=2,1 \leq \mathrm{i} \leq \mathrm{t}
\end{aligned}
$$

Then $v_{f}(0)=t, v_{f}(1)=t+1, v_{f}(2)=t$

$$
e_{f}(0)=t, e_{f}(1)=t, e_{f}(2)=t .
$$

Therefore, $\left|\mathrm{v}_{\mathrm{f}}(\mathrm{i})-\mathrm{v}_{\mathrm{f}}(\mathrm{j})\right| \leq 1$ and $\left|\mathrm{e}_{\mathrm{f}}(\mathrm{i})-\mathrm{e}_{\mathrm{f}}(\mathrm{j})\right|$ for all $i, j \in\{0,1,2\}$.

Hence $P_{n}$ is a root cube mean Cordial graph.
Case (iii) $\mathrm{n} \equiv 2(\bmod 3)$

Let $\mathrm{n}=3 \mathrm{t}+2$. Define $\mathrm{f}\left(\mathrm{u}_{\mathrm{i}}\right)=0,1 \leq \mathrm{i} \leq \mathrm{t}+1$

$$
\mathrm{f}\left(\mathrm{u}_{\mathrm{t}+1+\mathrm{i}}\right)=1,1 \leq \mathrm{i} \leq \mathrm{t}+1
$$

$$
\mathrm{f}\left(\mathrm{u}_{2 \mathrm{t}+2+\mathrm{i}}\right)=2,1 \leq \mathrm{i} \leq \mathrm{t}
$$

Then $\mathrm{v}_{\mathrm{f}}(0)=\mathrm{t}+1, \mathrm{v}_{\mathrm{f}}(1)=\mathrm{t}+1, \mathrm{v}_{\mathrm{f}}(2)=\mathrm{t}$

$$
e_{\mathrm{f}}(0)=t+1, e_{\mathrm{f}}(1)=t, e_{\mathrm{f}}(2)=t \text {. }
$$

Therefore, $\left|v_{f}(i)-v_{f}(j)\right| \leq 1$ and $\left|e_{f}(i)-e_{f}(j)\right| \leq 1$ for all $i, j \in\{0,1,2\}$.

Hence $P_{n}$ is a root cube mean Cordial graph.

Example 2.4: Root cube mean cordial labeling of the path $\mathrm{P} 9, \mathrm{P}_{10}, \mathrm{P}_{11}$ is given below.

\section{Theorem: 2.5}

The cycle $C_{n}$ is a root cube mean cordial graph if $\mathrm{n} \equiv 1(\bmod 3)$ and $\mathrm{n} \equiv 2(\bmod 3)$

\section{Proof:}

Let $C_{n}$ be the cycle $u_{1} u_{2} \ldots . u_{n} u_{1}$ where $\left|\mathrm{V}\left(\mathrm{C}_{\mathrm{n}}\right)\right|=\mathrm{n}$ and $\left|\mathrm{E}\left(\mathrm{C}_{\mathrm{n}}\right)\right|=\mathrm{n}$.

Define $f: V\left(C_{n}\right) \rightarrow\{0,1,2\}$ as follows.

Case (i) $\mathrm{n} \equiv 1(\bmod 3)$

Let $\mathrm{n}=3 \mathrm{t}+1$. Define $\mathrm{f}\left(\mathrm{u}_{\mathrm{i}}\right)=0,1 \leq \mathrm{i} \leq \mathrm{t}$

$$
\begin{aligned}
& \mathrm{f}\left(\mathrm{u}_{\mathrm{t}+\mathrm{i}}\right)=1,1 \leq \mathrm{i} \leq \mathrm{t}+1 \\
& \mathrm{f}\left(\mathrm{u}_{2 \mathrm{t}+\mathrm{i}+1}\right)=2,1 \leq \mathrm{i} \leq \mathrm{t}
\end{aligned}
$$

Then $v_{\mathrm{f}}(\mathrm{o})=\mathrm{t}, \mathrm{v}_{\mathrm{f}}(1)=\mathrm{t}+1, \mathrm{v}_{\mathrm{f}}(2)=\mathrm{t}$

$$
\mathrm{e}_{\mathrm{f}}(\mathrm{o})=\mathrm{t}+1, \mathrm{e}_{\mathrm{f}}(1)=\mathrm{t}, \mathrm{e}_{\mathrm{f}}(2)=\mathrm{t} \text {. }
$$

Therefore, $\left|v_{f}(i)-v_{f}(j)\right| \leq 1$ and $\left|e_{f}(i)-e_{f}(j)\right| \leq 1$ for all $i, j \in\{0,1,2\}$.

Hence $\mathrm{C}_{\mathrm{n}}$ is a root cube mean cordial graph.

Case (ii) $\mathrm{n} \equiv 2(\bmod 3)$

Let $n=3 t+2$. Define $f\left(u_{i}\right)=0,1 \leq i \leq t$

$$
\mathrm{f}\left(\mathrm{u}_{\mathrm{t}+\mathrm{i}}\right)=1,1 \leq \mathrm{i} \leq \mathrm{t}+1
$$




$$
\begin{gathered}
\mathrm{f}\left(\mathrm{u}_{2+1+\mathrm{i}}\right)=2,1 \leq \mathrm{i} \leq \mathrm{t} \\
\mathrm{f}\left(\mathrm{u}_{2}\right)=0 .
\end{gathered}
$$

Then $v_{f}(0)=t, v_{f}(1)=t+1, v_{f}(2)=t+1$

$$
e_{f}(0)=t+1, e_{f}(1)=t, e_{f}(2)=t+1 \text {. }
$$

Therefore, $\left|v_{f}(i)-v_{f}(j)\right| \leq 1$

for all $i, j \in\{0,1,2\}$ and $\left|e_{f}(i)-e_{f}(j)\right| \leq 1$

for all $i, j \in\{0,1,2\}$.

Hence $\mathrm{C}_{a}$ is a root cube mean cordial graph.

\section{Remarks: $\mathbf{2 . 6}$}

The cycle $\mathrm{C}_{a}$ is not a root cube mean graph if

$\mathrm{n} \equiv 0(\bmod 3)$

\section{Proof:}

Let $\mathrm{C}_{\mathrm{a}}$ be the cycle $\mathrm{u}_{1} \mathrm{u}_{2} \ldots \mathrm{u}_{\mathrm{\Omega}} \mathrm{u}_{1}$ where $\left|\mathrm{V}\left(\mathrm{C}_{\mathrm{a}}\right)\right|=\mathrm{n}$ and $\left|\mathrm{E}\left(\mathrm{C}_{2}\right)\right|=\mathrm{n}$.

Define $f: V\left(C_{2}\right) \rightarrow\{0,1,2\}$

Consider $\mathrm{n} \equiv 0(\bmod 3)$.

Suppose the cycle $\mathrm{C}_{\mathrm{a}}$ admits a root cube mean cordial graph if $\mathrm{n} \equiv(\bmod 3)$.

Let $\mathrm{n}=3 \mathrm{t}$.

Then $v_{f}(0)=t, v_{f}(1)=t, v_{f}(2)=t$ and $e_{f}(0)=t$, $e_{f}(1)=t-1, e_{f}(2)=t+1$. Therefore, $\left|v_{f}(i)-v_{f}(j)\right| \leq$ 1 for all $i, j \in\{0,1,2\}$ but $\left|e_{f}(i)-e_{f}(j)\right| \leq 1$ for all $i, j \in\{0,1,2\}$ not satisfied, Since $\mid e_{f}(0)-$ $e_{f}(1) \mid>1$, which is a contradiction.

$\therefore$ Our assumption is wrong. Hence $\mathrm{C}_{a}$ is not root cube mean cordial graph.
Example 2.7: Root cube mean cordial labeling of the cycle $\mathrm{C}_{4}$ and $\mathrm{C}_{5}$ is given below:

Theorem 2.8 The star $\mathrm{K}_{1, \text { is a }}$ a root cube mean cordial graph.

Proof :

Let $K_{1, \text { be }}$ the star with $n+1$ vertices and $n$ edges. Let $\mathrm{V}\left(\mathrm{K}_{\mathrm{la}}\right)=\left\{\mathrm{u}, \mathrm{u}_{\mathrm{i}}: 1 \leq \mathrm{i} \leq \mathrm{n}\right\}$ and $E\left(K_{1 @}\right)=\left\{u u_{i}: 1 \leq i \leq n\right\}$. Let $u$ be the centre of $\mathrm{K}_{\mathrm{l}, \mathrm{a}}$. Define $\mathrm{f}: \mathrm{V}\left(\mathrm{K}_{\mathrm{la}}\right) \rightarrow\{0,1,2\}$ as follows. Let $\mathrm{f}(\mathrm{u})=1$.

Case $(i) \mathrm{n} \equiv 0(\bmod 3)$

let $\mathrm{n}=3 \mathrm{t}$. Define $\mathrm{f}\left(\mathrm{u}_{\mathrm{i}}\right)=0,1 \leq \mathrm{i} \leq \mathrm{t}$

$$
\begin{aligned}
& \mathrm{f}\left(\mathrm{u}_{\mathrm{+}+\mathrm{i}}\right)=1,1 \leq \mathrm{i} \leq \mathrm{t} \\
& \mathrm{f}\left(\mathrm{u}_{2++i}\right)=2,1 \leq \mathrm{i} \leq \mathrm{t}
\end{aligned}
$$

Then $v_{f}(0)=t, v_{f}(1)=t+1, v_{f}(2)=t$ and

$$
e_{f}(0)=t, e_{f}(1)=t, e_{f}(2)=t .
$$

Therefore, $\left|v_{f}(i)-v_{f}(j)\right| \leq 1$ and

$$
e_{f}(i)-e_{f}(j) \mid \leq 1 \text { for all } i, j \in\{0,1,2\} \text {. }
$$

Hence $\mathrm{K}_{1} \_\mathrm{is}$ a root cube mean cordial graph

Case (ii) $\mathrm{n} \equiv 1(\bmod 3)$

Let $\mathrm{n}=3 \mathrm{t}+1$. Define $\mathrm{f}\left(\mathrm{u}_{\mathrm{i}}\right)=0,1 \leq \mathrm{i} \leq \mathrm{t}+1$

$$
\begin{aligned}
& \mathrm{f}\left(\mathrm{u}_{\mathrm{l}+1+\mathrm{i}}\right)=1,1 \leq \mathrm{i} \leq \mathrm{t} \\
& \mathrm{f}\left(\mathrm{u}_{2++1+\mathrm{i}}\right)=2,1 \leq \mathrm{i} \leq \mathrm{t}
\end{aligned}
$$

Then $v_{f}(0)=t+1, v_{f}(1)=t+1, v_{f}(2)=t$ and $e_{f}(0)=t+1, e_{f}(1)=t, e_{f}(2)=t$.

Therefore, $\left|v_{f}(i)-v_{f}(j)\right| \leq 1$ and $\left|e_{f}(i)-e_{f}(j)\right| \leq$ 1 for all $i, j \in\{0,1,2\}$.

Hence $\mathrm{K}_{\mathrm{l}, \Omega}$ is a root cube mean cordial graph Case $($ iii) $\mathrm{n} \equiv 2(\bmod 3)$

Let $\mathrm{n}=3 \mathrm{t}+2$. Define $\mathrm{f}\left(\mathrm{u}_{\mathrm{i}}\right)=0,1 \leq \mathrm{i} \leq \mathrm{t}+1$

$$
\begin{aligned}
& \mathrm{f}\left(\mathrm{u}_{\mathrm{t}+1+\mathrm{i}}\right)=1,1 \leq \mathrm{i} \leq \mathrm{t} \\
& \mathrm{f}\left(\mathrm{u}_{2 \mathrm{t}+1+\mathrm{i}}\right)=2,1 \leq \mathrm{i} \leq \mathrm{t}+1
\end{aligned}
$$

Then $v_{f}(0)=t+1, v_{f}(1)=t+1, v_{f}(2)=t+1$

$$
e_{f}(0)=t+1, e_{f}(1)=t, e_{f}(2)=t+1 \text {. }
$$

Therefore, $\left|v_{f}(i)-v_{f}(j)\right| \leq 1$ and $\left|e_{f}(i)-e_{f}(j)\right| \leq$ 1 for all $i, j \in\{0,1,2\}$.

Hence $\mathrm{K}_{1, \text { is }}$ a root cube mean cordial graph 
Example 2.9. Root cube mean cordial labeling of the star $\mathrm{K}_{1,7}$ is given below

\section{Theorem 2. 10}

Bistar graph $\mathrm{B}_{\mathrm{n}, \mathrm{n}}$ is a root cube mean cordial graph

\section{Proof :}

Let $B_{n, n}$ be the Bistar graph with vertex $s$ $\mathrm{V}\left(\mathrm{B}_{\mathrm{n}, \mathrm{n}}\right)=\left\{\mathrm{u}, \mathrm{v}, \mathrm{u}_{\mathrm{s}}, \mathrm{v}_{\mathrm{i}}: 1 \leq \mathrm{i} \leq \mathrm{n}\right\}$ and edges $\mathrm{E}\left(\mathrm{B}_{\mathrm{n}, \mathrm{n}}\right)$ $\left\{\mathrm{uv}, \mathrm{uu}_{\mathrm{s}}, \mathrm{vv}_{\mathrm{i}}: 1 \leq \mathrm{i} \leq \mathrm{n}\right\}$.

Define $\mathrm{f}: \mathrm{V}\left(\mathrm{B}_{\mathrm{n}, \mathrm{g}}\right) \rightarrow\{0,1,2\}$ as follows.

Let $\mathrm{f}(\mathrm{u})=1, \mathrm{f}(\mathrm{v})=1$.

\section{Case (i)}

$\mathrm{n} \equiv 2(\bmod 3)$. Let $\mathrm{n}=3 \mathrm{t}-1$

Define $\mathrm{f}\left(\mathrm{u}_{\mathrm{i}}\right)=0,1 \leq \mathrm{i} \leq 2 \mathrm{t}$

$$
\begin{aligned}
& \mathrm{f}\left(\mathrm{u}_{\mathrm{i}}\right)=1,2 \mathrm{t}+1 \leq \mathrm{i} \leq \mathrm{n} \\
& \mathrm{f}\left(\mathrm{v}_{\mathrm{i}}\right)=2,1 \leq \mathrm{i} \leq 2 \mathrm{t} \\
& \mathrm{f}\left(\mathrm{v}_{\mathrm{i}}\right)=1,2 \mathrm{t}+1 \leq \mathrm{i} \leq \mathrm{n}
\end{aligned}
$$

Then $\mathrm{v}_{f}(0)=\mathrm{v}_{f}(1)=\mathrm{v}_{f}(2)=2 \mathrm{t}$

$$
e_{f}(0)=2 t, e_{f}(1)=2 t-1, e_{f}(2)=2 t .
$$

Therefore, $\left|v_{\mathrm{f}}(\mathrm{i})-\mathrm{v}_{\mathrm{f}}(\mathrm{j})\right| \leq 1 \forall \mathrm{i}, \mathrm{j} \in\{0,1,2\}$ and $\left|\mathrm{e}_{(}(\mathrm{i})-\mathrm{e}_{\mathrm{f}}(\mathrm{j})\right| \leq 1 \forall \mathrm{i}, \mathrm{j} \in\{0,1,2\}$

Case (ii) $\mathrm{n} \equiv 0(\bmod 3)$, let $\mathrm{n}=3 \mathrm{t}$

Define $\mathrm{f}\left(\mathrm{u}_{\mathrm{i}}\right)=0,1 \leq \mathrm{i} \leq \mathrm{t}+1$

$$
\begin{aligned}
& \mathrm{f}\left(\mathrm{u}_{\mathrm{i}}\right)=1, \mathrm{t}+2 \leq \mathrm{i} \leq \mathrm{n} \\
& \mathrm{f}\left(\mathrm{v}_{\mathrm{i}}\right)=2,1 \leq \mathrm{i} \leq 2 \mathrm{t} \\
& \mathrm{f}\left(\mathrm{v}_{\mathrm{i}}\right)=0,2 \mathrm{t}+1 \leq \mathrm{i} \leq \mathrm{n}
\end{aligned}
$$

Then $\mathrm{v}_{f}(0)=\mathrm{v}_{\mathrm{f}}(1)=2 \mathrm{t}+1, \mathrm{v}_{\mathrm{f}}(2)=2 \mathrm{t}$

$e_{f}(0)=2 t+1, e_{f}(1)=2 t, e_{f}(2)=2 t$

Therefore $\left|\mathrm{v}_{\mathrm{f}}(\mathrm{i})-\mathrm{v}_{\mathrm{f}}(\mathrm{j})\right| \leq 1 \forall \mathrm{i}, \mathrm{j} \in\{0,1,2\}$

$\left|e_{(}(\mathrm{i})-\mathrm{e}_{\mathrm{f}}(\mathrm{j})\right| \leq 1 \forall \mathrm{i}, \mathrm{j} \in\{0,1,2\}$

\section{Case (iii)}

$\mathrm{n} \equiv 1(\bmod 3)$, let $\mathrm{n}=3 \mathrm{t}+1$
Define $f\left(u_{i}\right)=0,1 \leq i \leq 2 t+1$

$$
\begin{aligned}
& \mathrm{f}\left(\mathrm{u}_{\mathrm{i}}\right)=1,2 \mathrm{t}+2 \leq \mathrm{i} \leq \mathrm{n} \\
& \mathrm{f}\left(\mathrm{v}_{\mathrm{i}}\right)=2,1 \leq \mathrm{i} \leq 2 \mathrm{t}+1 \\
& \mathrm{f}\left(\mathrm{v}_{\mathrm{i}}\right)=1,2 \mathrm{t}+2 \leq \mathrm{i} \leq \mathrm{n}
\end{aligned}
$$

Then $\mathrm{v}_{\mathrm{f}}(0)=2 \mathrm{t}+1, \mathrm{v}_{\mathrm{f}}(1)=2 \mathrm{t}+2, \mathrm{v}_{\mathrm{f}}(2)=2 \mathrm{t}+1$ $e_{f}(0)=e_{f}(1)=e_{k}(2)=2 t+1$

Therefore $\left|\mathrm{v}_{\mathrm{f}}(\mathrm{i})-\mathrm{v}_{\mathrm{f}}(\mathrm{j})\right| \leq 1 \forall \mathrm{i}, \mathrm{j} \in\{0,1,2\}$

$\left|e_{f}(\mathrm{i})-\mathrm{e}_{(}(\mathrm{j})\right| \leq 1 \forall \mathrm{i}, \mathrm{j} \in\{0,1,2\}$

From all the above cases, we have $B_{n, n}$ is a root cube mean cordial graph.

\section{Theorem 2. 11.}

The complete graph $\mathrm{K}_{n}$ is not a root cube mean cordial graph for any $n>3$.

\section{Proof:}

Let $\mathrm{G}$ be the complete graph $\mathrm{K}_{\mathrm{n}}$ with $\mathrm{n}$ vertices and $n(n-1) / 2$ edges. Clearly $K_{1}, K_{2}, K_{9}$ are root cube mean cordial graphs. Let us check for the complete graphs $K_{n}, n>3$

Define $\mathrm{f}: \mathrm{V}\left(\mathrm{K}_{\mathrm{n}}\right) \rightarrow\{0,1,2\}$ as follows :

Case (i) $\mathrm{n} \equiv 0(\bmod 3)$

Let $n=3 t$

Then $v_{f}(0)=v_{f}(1)=v_{f}(2)=t$

$e_{f}(0)=\left(\begin{array}{l}t \\ 2\end{array}\right)+t^{2}$

$e_{f}(1)=\left(\begin{array}{l}t \\ 2\end{array}\right)$,

$\mathrm{e}_{\mathrm{f}}(2)=\left(\begin{array}{l}\mathrm{t} \\ 2\end{array}\right) \mathrm{t}^{2}+\mathrm{t}^{2}$, a contradiction, 
since $\left|e_{f}(\mathrm{i})-e_{f}(\mathrm{j})\right|>1$

Case (ii) $\mathrm{n} \equiv 1(\bmod 3)$

Let $\mathrm{n}=3 \mathrm{t}+1$

Subcase (i) If $\mathrm{v}_{\mathrm{f}}(0)=\mathrm{t}+1, \mathrm{v}_{\mathrm{f}}(1)=\mathrm{v}_{\mathrm{f}}(2)=\mathrm{t}$

$$
\begin{aligned}
& \text { then } e_{f}(0)=\left(\begin{array}{c}
t+1 \\
2
\end{array}\right)+t(t+1) \\
& e_{f}(1)=\left(\begin{array}{c}
t+1 \\
2
\end{array}\right)-t \\
& e_{f}(2)=\left(\begin{array}{l}
t \\
2
\end{array}\right)+t(t+1)+t^{2}, \text { a contradiction, } \\
& \text { since }\left|e_{f}(i)-e_{i}(j)\right|>1
\end{aligned}
$$

Subcase (ii) If $\mathrm{v}_{\mathrm{f}}(0)=\mathrm{v}_{\mathrm{f}}(2)=\mathrm{t}, \mathrm{v}_{\mathrm{f}}(1)=\mathrm{t}+1$

then $e_{f}(0)=\left(\begin{array}{c}t+1 \\ 2\end{array}\right)+t^{2}$

$$
\begin{aligned}
& e_{f}(1)=\left(\begin{array}{c}
t+1 \\
2
\end{array}\right) \\
& e_{f}(2)=\left(\begin{array}{l}
t \\
2
\end{array}\right)+t(t+1)+t^{2}, \text { a contradiction, }
\end{aligned}
$$

since $\left|e_{f}(\mathrm{i})-e_{f}(\mathrm{j})\right|>1$

Subcase (iii) If $\mathrm{v}_{\mathrm{f}}(0)=\mathrm{v}_{\mathrm{f}}(1)=\mathrm{t}, \mathrm{v}_{\mathrm{f}}(2)=\mathrm{t}+1$

then $e_{f}(0)=\left(\begin{array}{l}t \\ 2\end{array}\right)+t^{2}$

$$
\begin{aligned}
& e_{f}(1)=\left(\begin{array}{c}
t+1 \\
2
\end{array}\right) \square t \\
& e_{f}(2)=\left(\begin{array}{c}
t+1 \\
2
\end{array}\right)+t(t+1)+t(t+1),
\end{aligned}
$$

a contradiction

$$
\text { Case (iii) } n \equiv 2(\bmod 3)
$$

Let $\mathrm{n}=3 \mathrm{t}+2$

Subcase (i) If $v_{f}(0)=t, v_{f}(1)=v_{f}(2)=t+1$ then $e_{f}(0)=\left(\begin{array}{c}t+1 \\ 2\end{array}\right)+t^{2}$

$$
e_{f}(1)=\left(\begin{array}{c}
t+1 \\
2
\end{array}\right)
$$

$$
e_{N}(2)=\left(\begin{array}{c}
t+2 \\
2
\end{array}\right)+t(t+1)+t(t+1)
$$

a contradiction, since $\left|e_{f}(\mathrm{i})-e_{f}(\mathrm{j})\right|>1$

Subcase (ii) If $v_{f}(1)=t, v_{f}(0)=v_{f}(2)=t+1$

$$
\text { then } \begin{aligned}
e_{f}(0) & =\left(\begin{array}{c}
t+1 \\
2
\end{array}\right)+t(t+1) \\
e_{f}(1) & =\left(\begin{array}{c}
t+1 \\
2
\end{array}\right)-t
\end{aligned}
$$

$$
e_{f}(2)=\left(\begin{array}{c}
t+2 \\
2
\end{array}\right)+t(t+1)+t(t+1),
$$

a contradiction, since $\left|e_{i}(i)-e_{i}(j)\right|>1$

Subcase (iii) If $v_{f}(2)=t, v_{f}(0)=v_{f}(1)=t+1$

$$
\text { then } \begin{aligned}
e_{f}(0) & =\left(\begin{array}{c}
t+1 \\
2
\end{array}\right)+(t+1)^{2} \\
e_{k}(1) & =\left(\begin{array}{c}
t+1 \\
2
\end{array}\right)
\end{aligned}
$$

$$
\mathrm{e}_{\mathfrak{f}}(2)=\left(\begin{array}{c}
\mathrm{t}+1 \\
2
\end{array}\right)+\mathrm{t}(\mathrm{t}+1)+\mathrm{t}^{2}, \text { a contradiction, }
$$

since $\left|e_{i}(\mathrm{i})-e_{i}(\mathrm{j})\right|>1$

From all the above cases, $\mathrm{K}_{\mathrm{n}}$ is not a root cube mean cordial graph.

\section{Theorem 2. 12}

The complete bipartite graph $\mathrm{K}_{2, n}$ is not root cube mean cordial for any $n>2$.

\section{Proof:}

Let $\mathrm{V}\left(\mathrm{K}_{2, \mathrm{n}}\right)=\mathrm{A} \cup \mathrm{B}$, where $\mathrm{A}=\{\mathrm{u}, \mathrm{v}\}$ 
and $B=\left\{u_{1}, u_{2}, \ldots, u_{n}\right\}$.Then $E\left(K_{2 a}\right)=\left\{u_{i}, v u_{i}: 1 \leq i\right.$

$\leq \mathrm{n}\}$. Clearly $\mathrm{K}_{2,1}$ and $\mathrm{K}_{2,2}$ are root cubemean cordial

graph.Assume that $\mathrm{n}>2$. Here $\mathrm{K}_{2, \mathrm{~g}}$ has $2+\mathrm{n}=\mathrm{m}$

(say) vertices and $2 n=2(m \square 2)$ edges.

Case (i) $\mathrm{m} \equiv 0(\bmod 3)$. Let $\mathrm{m}=3 \mathrm{t}$

Without violating the vertex label differences assign labels to the vertices $v_{f}(0)=v_{f}(1)=v_{f}(2)=t$, we have the following possibilities.

\section{Subcase (a)}

Let $f(u)=0, f(v)=0$. Then $e_{f}(0)=4 t-4, e_{f}(1)=0$, $e_{f}(2)=2 t$.

\section{Subcase (b)}

Let $f(u)=0, f(v)=1$. Then $e_{f}(0)=3(t-1), e_{f}(1)=$ $\mathrm{t}-1, \mathrm{e}_{\mathrm{f}}(2)=2 \mathrm{t}$

\section{Subcase (c)}

Let $\mathrm{f}(\mathrm{u})=1, \mathrm{f}(\mathrm{v})=0$. Then $\mathrm{e}_{\mathrm{f}}(0)=3(\mathrm{t}-1)$,

$e_{f}(1)=t-1, e_{f}(2)=2 t$

\section{Subcase (d)}

Let $f(u)=0, f(v)=2$. Then $e_{f}(0)=2 t-1$,

$$
e_{f}(1)=0, e_{f}(2)=4 t-3
$$

\section{Subcase (e)}

Let $f(u)=2, f(v)=0$. Then $e_{f}(0)=2 t-1$,

$e_{f}(1)=0, e_{f}(2)=4 t-3$

\section{Subcase (f)}

Let $f(u)=1, f(v)=1$. Then $e_{f}(0)=2 t$, $e_{f}(1)=2 t-4, e_{f}(2)=2 t$

\section{Subcase (g)}

Let $f(u)=1, f(v)=2$. Then $e_{f}(0)=t$,

$e_{f}(1)=t-1, e_{f}(2)=4 t-3$

\section{Subcase (h)}

Let $\mathrm{f}(\mathrm{u})=2, \mathrm{f}(\mathrm{v})=1$. Then $\mathrm{e}_{\mathrm{f}}(0)=\mathrm{t}$,

$e_{f}(1)=t-1, e_{f}(2)=4 t-3$

Subcase (i)

Let $f(u)=2, f(v)=2$.

Then $e_{f}(0)=0, e_{f}(1)=0, e_{f}(2)=6 t-4$

All the above subcases, the condition that $\mid e_{f}(i)=$ $\mathrm{e}_{\mathrm{f}}(\mathrm{j}) \mid \leq 1 \forall \mathrm{i}, \mathrm{j}$ is not satisfied. Hence we can see that $\mathrm{K}_{2,0}$ is not root cube mean cordial.

Thus in the following cases, we consider only the subcases in which the vertices $u$ and $v$ are having labels 1 and 2 respectively.

\section{Case (ii)}

$\mathrm{m} \equiv 1(\bmod 3)$. Let $\mathrm{m}=3 \mathrm{t}+1$. Suppose $\mathrm{f}(\mathrm{u})=1$ and $f(v)=2$

\section{Subcase (a)}

If $v_{f}(0)=t+1, v_{f}(1)=v_{f}(2)=t$. Then $e_{f}(0)=t+1$, $e_{f}(1)=t-1, e_{f}(2)=4 t-2$

\section{Subcase (b)}

If $v_{f}(1)=t+1, v_{f}(0)=v_{f}(2)=t$. Then $e_{f}(0)=t$, $e_{f}(1)=t, e_{f}(2)=4 t-2$

\section{Subcase (c)}

If $v_{f}(2)=t+1, v_{f}(0)=v_{f}(1)=t$. Then $e_{f}(0)=t$, $e_{f}(1)=t-1, e_{f}(2)=4 t-1$ 


\section{Case (iii)}

$\mathrm{m} \equiv 2(\bmod 3)$, Let $m=3 t+2$. Suppose $f(u)=1$ and $f(v)=2$

\section{Subcase (a)}

If $\mathrm{v}_{\mathrm{f}}(0)=\mathrm{v}_{\mathrm{f}}(1)=\mathrm{t}+1, \mathrm{v}_{\mathrm{f}}(2)=\mathrm{t}$. Then $\mathrm{e}_{\mathrm{f}}(0)=\mathrm{t}+1$, $e_{f}(1)=t, e_{f}(2)=4 t-1$

\section{Subcase (b)}

If $v_{f}(0)=v_{f}(2)=t+1, v_{f}(1)=t$. Then $e_{f}(0)=t+1$, $e_{f}(1)=t-1, e_{f}(2)=4 t$.

\section{Subcase (c)}

If $v_{f}(0)=t, v_{f}(1)=v_{f}(2)=t+1$. Then $e_{f}(0)=t$, $\mathrm{e}_{\mathrm{f}}(1)=\mathrm{t}, \quad \mathrm{e}_{\mathrm{f}}(2)=4 \mathrm{t} . f$ does not satisfies the conditions $\left|v_{f}(\mathrm{i})-v_{f}(j)\right| \leq 1$ and $\left|e_{f}(i)-e_{f}(j)\right| \leq$ 1 , for $i, j \in\{0,1,2\}$. Hence $K_{2 s}$ is not root cube mean cordial graph.

Figure 1: A root cube mean cordial graph

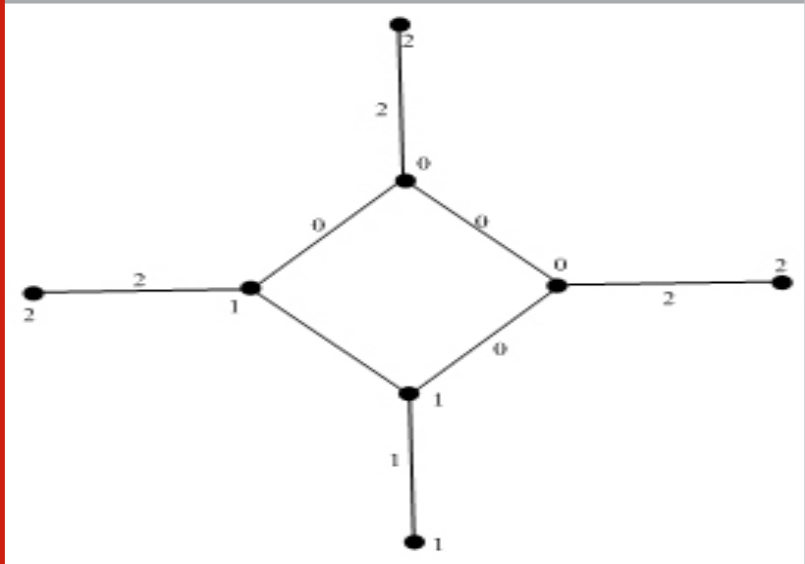

Figure 1.1

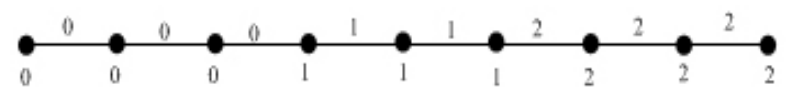

Figure 1.2

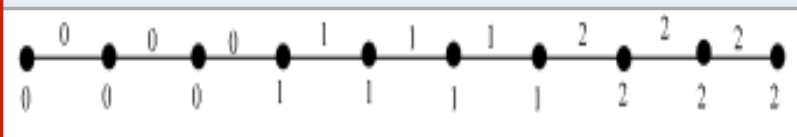

Figure 1.3

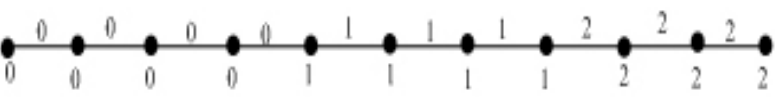

Figure: 4

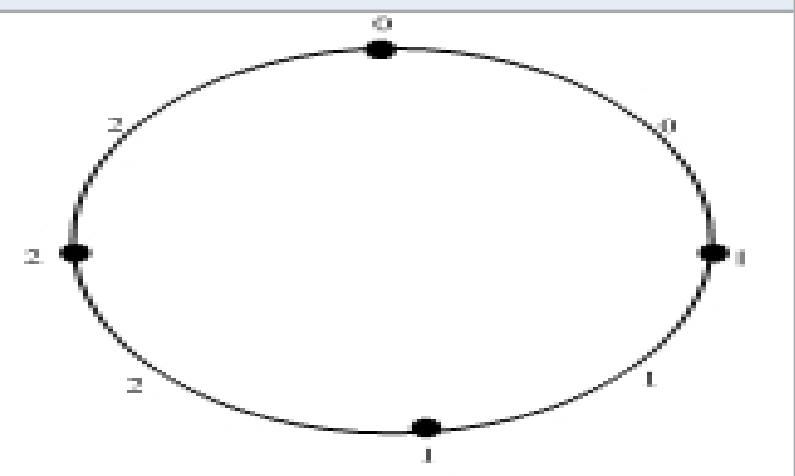

Figure: 5

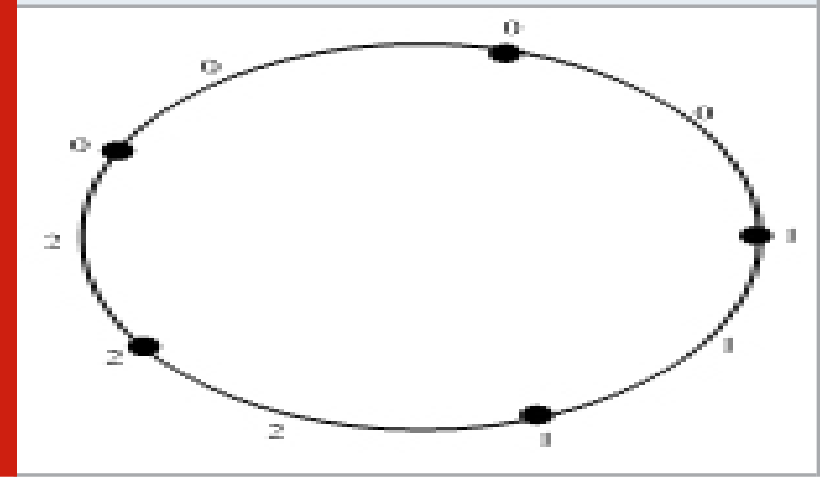

Figure: 6

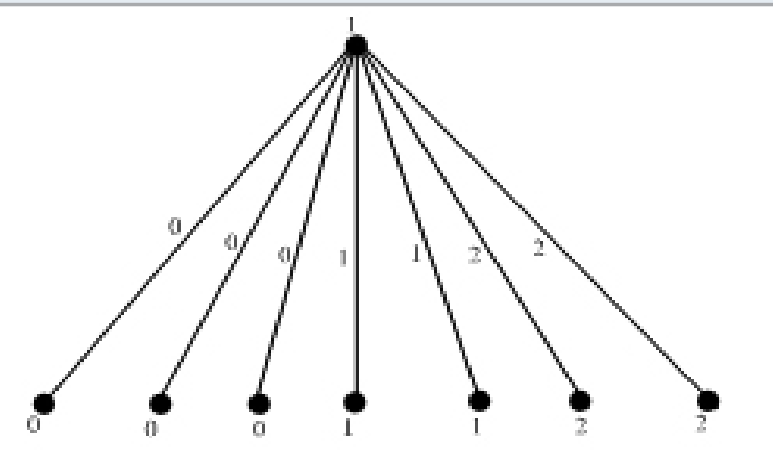

\section{CONCLUSION}

In this paper root cube mean cordiality of some standard graphs such as cycle, path, star, complete graph, complete bipartite graph and bistar are studied.

\section{REFERENCES}

Bondy, J.A., Murthy, U.S.R., (1976). Graph Theory with Applications, Newyork, North Holland,

Cahit, I., (1987). Cordial Graphs: A weaker Version of graceful and harmonious graphs, Ars. Combinatoria, Vol 23, No. 3, pp. 201-207.

Gallian, J.A., (2011). A. Dynamic Survey of Graph Labeling, Vol.18

Harary, F., (1988). Graph Theory, Narosa Publishing 
House Reading, New Delhi.

Ponraj, R., Sivakumar, M., ( 2012). Mean Cordial labeling of graphs, Open Journal of Discrete Mathematics, Vol.2, No.4, pp 145.

Sandhya, S.S., Somasundaram, S., Ponraj, R., (2012). Harmonic Mean Labeling of Some Cycle Related
Graphs. Int. Journal of Math. Analysis, Vol.6, No. 40, pp 1997-2005.

Sandhya, S.S., Somadundaram, S., (2013). Harmonic Mean Labeling for Some Special Graphs, International Journal of Mathematics Research, Vol.5, No.1, pp.5564. 